\title{
Review
}

\section{Adult Neurogenesis in Injury-Induced Self-Repair: Use It or Lose It}

\author{
Mei-Fang Cheng* \\ Department of Psychology, Rutgers, The State University of New Jersey, Newark, NJ, USA
}

\begin{abstract}
One of the most hotly pursued topics in neuroscience and therapeutic medicine is the use of stem cells in the adult brain. Growing in parallel to this emerging field is the recognition that the adult brain is indeed capable of generating new cells. While neurogenesis was understood to be restricted to a few areas, recent studies suggest that damage to the adult brain can trigger neurogenesis even in regions outside of these specific areas. This finding raises the possibility that neurons born in response to perturbation in the brain may be involved in the recovery of function in the damaged adult brain. The key is understanding how to cultivate these newborn cells, because they do not remain viable if they are not accepted into the damaged network of interconnected neurons which support specific functions. From a birth site, undifferentiated precursor cells or neurons undergo migration and differentiation. Many factors influence the safe journey of migrating cells and their survival after maturation at their destination.

This review will present evidence from ring dove studies that an activity-dependent mechanism underlies the survival of adult newborn neurons and establishment of their functionality. This evidence includes: [1] unique electrophysiological properties or specific connectivity associated with various type of neurons involved in ring dove coo behavior and reproductive function, [2] emergence of electrophysiological properties and specific projection neurons emanating from newborn neurons after hypothalamic lesion, and finally [3] collective behavioral analyses of social stimulations suggesting that sensorimotor events contribute to the integration of new neurons and reinstatement of function.
\end{abstract}

Keywords: Hypothalamus, adult neurogenesis, recovery of functions

\section{INTRODUCTION}

Advances in neuroscience have provided an understanding of how sensory events contribute to brain and bodily functions. The brain is endowed with unique sets of sensory and perceptual attributes that filter external stimuli to facilitate the stability and maintenance of the living organism. As interest in the study of aging has surged, particularly regarding

\footnotetext{
*Correspondence to: Mei-Fang Cheng, Rutgers University, Department of Psychology, 101 Warren St., Newark, NJ 07059, USA. Tel.: +1 973 353 5440/Ext.: 1828; Fax: +1 973353 5440/Ext.: 1171; E-mail: mfc@psychology.rutgers.edu.
}

the decline of bodily and brain functions, the old maxim of "use it or lose it" has been touted as a guiding principle for medical treatment and health advice [1]. The science behind this maxim, however, is still in its infancy. Some advances have been made in the study of peripheral systems. For example, animal studies have elucidated the physiological processes of healing after usage versus resting of injured limbs [2-5]. Findings that exercise significantly increases synovial fluid in osteoarthritisaffected horses [6,7] and that the role of synovial fluid is to reduce friction between the articular cartilage of synovial joints during movement in animals and 
humans [8] support the seemingly counterintuitive recommendation of exercise as a first-line treatment for managing arthritic joint pain in humans. Most studies, however, are correlational in nature, and in particular, studies validating the causal relationship between usage of neural circuits or cognitive function and the retention or recovery of function are far and few between.

In this paper, we will present how the same principle of usage operates in the cultivation of newborn neurons in the adult brain. Brain cells born (neurogenesis) after birth are conserved across species from crustaceans to higher vertebrates $[9,10]$. In phylogenetically more recent species including humans [10-13] neurogenesis appears to be limited to specific brain regions. The dentate gyrus of hippocampus and subventricular zone (SVZ) of olfactory bulb are two of the regions which are most characteristic of, and accepted as, neurogenic niches: that is, regions that normally incorporate new neurons throughout adulthood [14-16]. Functional neurogenesis exists in other less known regions, most notably, the dietresponsive neurogenic niche in median eminence regions [17-19]. Equally important are reports of low level regenerative activity in the neocortex, amygdala, and striatum of primates, the CA1 region of the hippocampus and in the nigra substantia of mice, and in striatal dopaminergic neurons [20-23], although some of these have been controversial [16, 24]. Interestingly, non-neurogenic precursor cells from outside of the dentate gyrus can differentiate into neurons specific to the region when transplanted into the neurogenic dentate gyrus $[16,25]$. This finding suggests the feasibility that non-neurogenic regions can recruit new neurons in the presence of the following two permissive factors.

(1) Neurogenic promoting factors. Selective evolutionary pressure in mammals may have restricted neurogenic activity to a few brain regions for specific normal functions. However, various form of growth factors appear to enable the brain to retain some capacity to support and incorporate new neurons. For example, in mice and rats, infusion of EGF (epidermal growth factor) after stroke promotes SVZ proliferation and migration to the damaged striatum [26, 27]. In rats intraventricular infusion of EGF and albumen or FGF2 (fibroblast growth factor2) after large forebrain ischemia resulted in the regeneration of striatal interneuron and hippocampal CA1pyramidal neurons, and reversed some functional deficit [28-30]. For a review of neurogenesis promoting factors, see Sohur et al. [16].
Regenerative activity in the non-or-low neurogenic regions can therefore be elevated by transforming the region into "permissive microenvironment" ${ }^{1}$ with the aid of various growth hormones.

(2) Cell death-induced chemical signals. The brain's reaction to damage includes chemical signals released by cell death which may serve to guide precursor cells from other brain regions; in particular, from neurogenic niches to the damaged region. Experimentally induced stroke in rats can trigger proliferation in SVZ and migration to non-neurogenic target sites [26-29]. Similarly, non-neurogenic brain regions such as the mouse neocortex, rat striatum, dove hypothalamus, and other regions can be induced to recruit neurons born from neurogenic regions following assault, [22, 31, 32]. Although the functionality of induced-new neurons following cell death has been documented in the normally neurogenic adult hippocampus and song nuclei HVC [10, $33,34]$, with the exception of hippocampal pyramidal neurons [30], a rigorous elucidation of the self-repair process (compensatory neurogenesis) in non-neurogenic brain regions has yet to be accomplished [35].

In birds, research interest in adult neurogenesis is closely related to understanding the birdsong system. Seasonal changes in adult neurogenesis in the HVC (high vocal center: a telencephalic structure for song production) region of canaries provides a rationale for linking seasonal song learning to seasonal changes in neurogenic activity. Supportive evidence can be found in zebra finch studies which show that the selective killing of HVC neurons which send projection to area RA (the nucleus robustus archistriatum) responsible for song output will trigger neuronal recruitment. The rate of induced neurogenesis and the recovery of the birds' ability to produce song coincide with new projections from HVC and area RA [37]. These findings have led to the notion that adult neurogenesis is limited to the song system of song birds [38]. In truth, adult neurogenesis is evolutionarily conserved for a variety of functions [17]. We chose the ring dove for the study of adult neurogenesis, because the hypothalamic system in the adult ring dove is non-neurogenic, as described further below. As a consequence, results may be generalized to other non-neurogenic regions. Furthermore, comparative analyses between birds and mammals can provide useful knowledge at a structural level.

\footnotetext{
1 "Permissive microenvironment" was first used in the article by Sohur et al. [16].
} 
For example, the precursor cells in the subventricular zone in rodents proliferate and migrate to the olfactory bulb, but in birds, migrate to a wider regions [10], importantly, similar mechanisms of cell death induced compensatory neurogenesis in non-neurogenic regions are active in both species. Comparative analyses at cellular and behavioral levels will clarify the applicability of various animal models for brain repair.

\section{RING DOVE MODEL FOR ADULT NEUROGENESIS AND RECOVERY OF FUNCTION}

The focus of our experimental series specifically addressed whether adult neurogenesis participates in the recovery of functionality after injury to the adult brain. Attempts to demonstrate functionality of adult newborn neurons beyond the hippocampus are difficult; the challenge is identifying a verifiable model of function and behavior that can be measured and used to assess newborn neurons in the adult system.

We chose the ventral medial hypothalamus $(\mathrm{VMH})^{2}$ of the ring dove as our model based on three selection criteria: [1] the specific physiology and behavior of the species is quantifiable and repeatable; [2] the basic neural circuitry of the physiological and behavioral events to be studied has been documented; and [3] the brain region to be studied is not normally neurogenic in the adult brain ${ }^{3}$. This last feature will allow us to generalize our findings to other brain regions that are not normally neurogenic. Importantly, although the VMH region is not neurogenic [38], it generates new neurons in the adult brain in response to cell loss after lesion [32]. Moreover, the neural mediation of key courtship behavior, the subject of these studies, is centrally congregated in the hypothalamus. This enables us to interpret neural events in the hypothalamus that are associated with functional changes attributable solely to this region and not eclipsed by the potential involvement of other intact regions of the brain.

\footnotetext{
${ }^{2}$ VMH includes VMN and PMH terms used in earlier studies activation of hypothalamus-pituitary-ovarian axis relies also on proper photo stimulation (Cheng 1976).

${ }^{3}$ Note: The specific regions of hypothalamic neurogenesis for energy control system in mice (Cheng, 2013) are outside of the region we studied.
}

\section{HYPOTHALAMIC SYSTEMS AND REPRODUCTIVE FUNCTION AND BEHAVIOR}

The ring dove is a monogamous species that pair bonds for life. The elaborate interactive courtship behavior between the partners, guided by "state reading" from each partner, helps to coordinate a predictable sequence culminating in egg-laying and rearing the young [39]. The courtship commences with male bow cooing (solicitation) to the female, followed by male nest cooing with oblique posture at the potential nest site. A critical milestone occurs when the female joins the male and together they perform duet nest cooing. This duet is followed by the female's solo nest coo, at which point the male leaves the site to hunt for nest materials and brings them back. Together the pair constructs the nest. The female continues cooing until she lays a clutch of two eggs, and then the male and female take turns incubating until the eggs hatch. Together, the pair raises their two squabs to 21 days of age. Adequate lighting is required for maintaining reproductive physiology, and lighting interacts with environmental factors in initiating courtship behavior which culminates in successful breeding [40]. The predictable sequence of these behavioral events during the breeding cycle provides us with a measurable reference from which to compare the nature and quantity of loss and reinstatement of functions after regeneration of new neurons. As it becomes clear in the following sections, the different types of neurons responsible for initiation of nest coo courtship behavior and reproduction are all conveniently located in the hypothalamus. These features make ring dove breeding behavior particularly suitable to the study of the role of adult born neurons in the recovery of function after brain injury.

Bilateral lesion in the ventral medial portion of the hypothalamus (VMH) abolishes the courtship behavior, specifically the nest coo behavior in both the male and female [41, 42]. Could destruction of some neurons in the VMH account for loss of courtship behavior and breeding cycle after electrolytic lesion in the region? We have previously identified three types of neurons in this region that are most related to breeding behavior: [1] Acoustic neurons, [2] Projection neurons, [3] GnRH (gonadotrophic release hormone) neurons. These are described further below.

(1) Acoustic neurons (coo-sensitive neurons). The nucleus ovoidalis (avian thalamic auditory relay) and its shell region project to the ventral hypothalamus 
[43] and transmit auditory cues, including coo signals to the VMH [44]. Recordings of neurons in the VMH receiving these inputs led us to identify neurons sensitive to gender-specific nest coo stimulation [45]. In females, the neurons sensitive to female nest coo stimulation (FC specific neurons), give a signature firing pattern when activated (Fig. 1); no other stimulation, including male nest coo or female nest coo played backward, can activate these FC specific neurons (Fig. 2).

To our delight, we noted a chain reaction of these FC specific neuronal firings, culminating in activation of the hypothalamus-pituitary -ovarian axis (HPO). Activation of FC specific neurons resulted in a threefold increase of luteinizing hormone (LH) output in the anterior pituitary of reproductively inexperienced females. This mechanism explains a behavioral observation made in 1986 that female coo promotes ovarian growth [46] and the female's persistent solo coos coincide with the exponential rise of LH prior to laying a clutch of two eggs $[47,48]$.

(2) Projection neurons. These neurons extend from VMH to the midbrain vocal control pathway, the pathway responsible for production of nest cooing [49].

(3) $\mathrm{GnRH}$ (gonadotrophic release hormone) neurons. These neurons control the hypothalamuspituitary-ovarian axis, and are mostly scattered in the preoptic area (POA, a poorly defined area with no clear border in the ring dove) just underneath the septomesencephalic tract (TSM) of anterior hypothalamus [50].

When electrolytic lesion is applied to the VMH region, the anterior hypothalamic region is invari-

\section{Unique Female Nest Coo Responsive Unit}

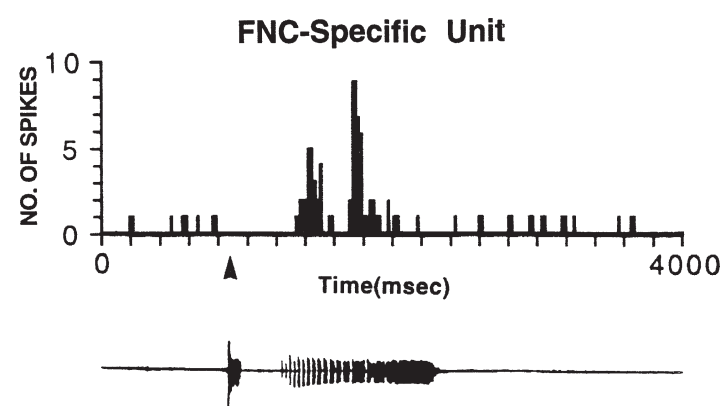

Fig. 1. Female Coo Specific Units 960127. Top, Histogram shows the unit's response to the female-nest-coo stimulus presented at $70+/-5$ bB SPL. Bottom, Computer amplitude display of the female nest coo. Courtesy of Cheng et al. J. of Neuroscience 1998; 18:5477-5489. ably included [49, 51, 52 ] consequently, GnRH neurons are affected. Destruction of these three neuron types (acoustic neurons, projection neurons and GnRH neurons) disrupts the sequence of courtship behavior and ultimately egg-laying. The loss of cooresponsive acoustic neurons deprives a bird of its ability (a) to respond to a partner's nest-coo and her own nest coo, (b) to utter its own nest-coo through projection neurons, and importantly for females, (c) to activate $\mathrm{FC}$ specific neurons which in turn trigger GnRH neurons to activate the HPO axis, resulting in elevated LH output for oviposition and ensuing egg-laying.

Given that VMH lesion triggers adult neurogenesis, precursor cells migrate from subventricular zone to VMH [32], we contemplated the possible role of adult neurogenesis in the recovery of behavior. In the 2006 and 2007 studies, we observed that recovery of nest coo behavior is possible after a recuperation period ( 8 weeks) during which time the lesioned birds were returned to their bonded mates $[49,51]$. Only later in this series of studies did we realize that the nature of this housing significantly impacts the rate of recovery, a topic we will return to later.

\section{SPECIFIC ELECTRICAL FIRING PATTERNS FROM NEWBORN NEURONS}

To better understand the neural events underlying the loss of function and eventual recovery in doves, we conducted a series of experiments in which we monitored the electrical and cellular changes in the region for 14 weeks after electrolytic lesion [52]. Most birds in this study were returned to stock cages after surgery. Immediately following lesion, the region was silent without any sign of electrical activity for up to 36 days, and then in a gradual, stepwise manner, electrical signal reappeared. The signal was initially just a blip, then a phasic pattern lacking the complete form of a discharge. Normal forms of spontaneous discharge did not appear reliably until after 13-14 weeks (Fig. 3).

During the first 50 days after lesion, newborn cells (cells labeled with a marker for detection of proliferating cells: BrdU+ cells), dominated the region and electrical signals from the region were without characteristic discharge. Newborn neurons (proliferating cells labeled with a marker for neuronal specific nuclear protein: $\mathrm{BrdU}+/ \mathrm{NeuN}+$ cells), gradually appeared at 60-70 days, a time when the number 
Electrophysiological response of a ring dove to different acoustic stimuli

A. Female Nest Coo

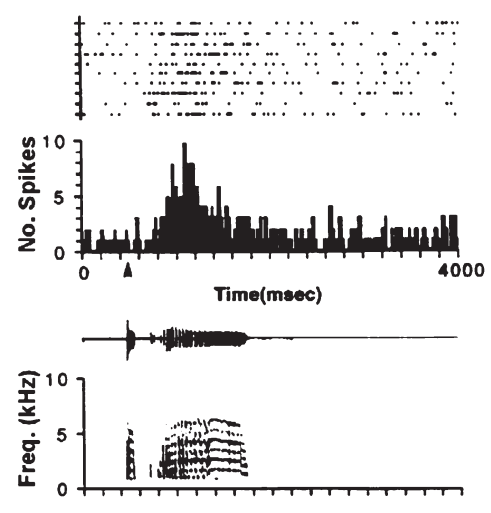

C. White Noise
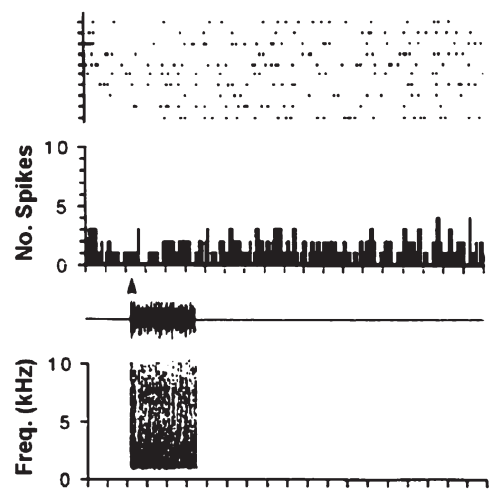

B. Reversed Female Nest Coo
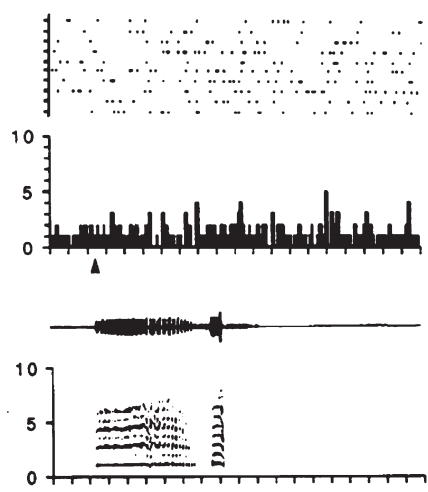

D. Male Nest Coo
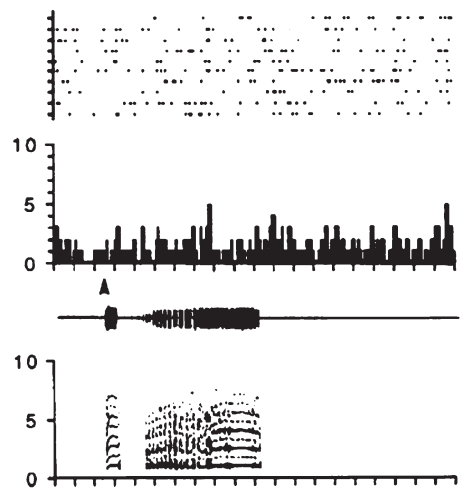

Cheng, Peng, Johnson, J Neurosc, 1998, 18:5477-5489

Fig. 2. Response of Female Nest-Coo Specific Units to Other Stimulations. Response of unit 960108 in the preoptic area of a female ring dove to different acoustic stimuli. $A$, The female nest coo as the stimulus; the activity of the unit increases significantly. Top, Dot raster plot showing 10 sweeps of the unit's response. Each dot represents one spike. Bin size is $20 \mathrm{msec}$. Middle, Histogram of the unit's response. Bottom, Computer amplitude display and spectrogram of the female nest coo: amplitude over time. $B$, Reversed female nest coo as the stimulus; no change in neuronal activity can be detected in the number of spikes over time or in the histogram representation. $C$, White noise as the stimulus: no change in neuronal activity. $D$, Male nest coo as the stimulus: no change in neuronal activity. All histograms represent the sum of 10 repetitions of the stimulus. All units are well isolated. In all figures, a closed triangle denotes the onset of the stimulus. Courtesy of Cheng et al. J Neurosci. 1998; 18: 5477-5489.

of abnormal spontaneous firing units in the region increased, of which about $1 \%$ responded to coo stimulation. Specific firing patterns evoked in response to a female's coo stimulation appeared at about the same time as the appearance of newborn neurons in the region. This coincidental timing suggests that the characteristic female coo-sensitive firing pattern $(F C$ specific pattern) likely originates from the newborn neurons. To test if this is the case, the position of the recording electrode was marked by iontophoresis of potamine sky blue dye (PSB). The dye at the tip of single unit recording micropipettes was dispensed at the end of each positive recording session. If cooresponsive new neurons give rise to the characteristic
FC-specific firing pattern, only coo-responsive new neurons should show PSB labeling. There were indeed triple-labeled cells (BrdU+/NeuN+/ PSB + ), suggesting that some newborn neurons had assumed the characteristic function of coo responsive auditory units [52]. It is worth emphasizing that our criteria for functional recovery was not limited to identifying the characteristic spontaneous firing pattern; we provide further evidence that the characteristic firing pattern is invoked only by specific coo (female coo) stimulation (Fig. 4).

We found that some of the newborn neurons send direct projections to the nucleus intercolliculus of the midbrain that controls vocal output in 


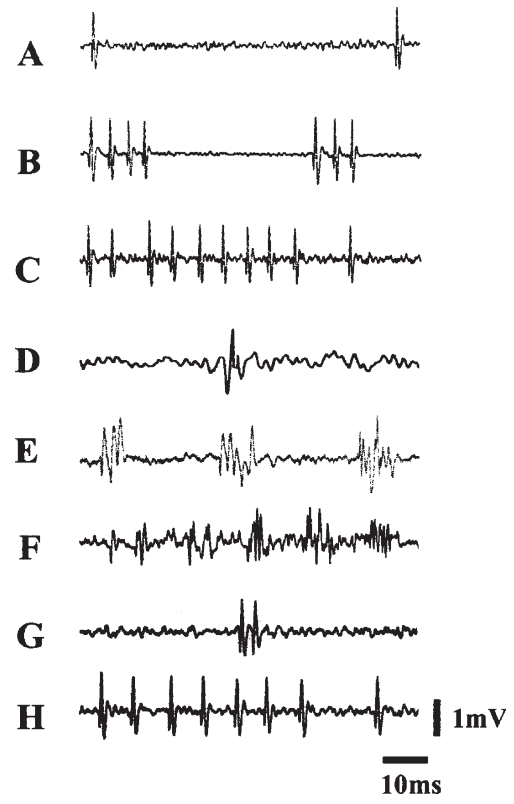

Fig. 3. Electrophysiological Activities Over the Course of 99 Days Postlesion. Samples of spontaneous electrical activity in VMN before and after lesions. (A), (B), and (C) were recorded from VMN before lesions: (A) A slow and irregular discharge pattern; (B) a phasic firing pattern; (C) a fast firing pattern. (D-H) were recorded after lesions: (D) was recorded at 42 days postlesion; (E) was recorded 64 days postlesion. Note that (D) and (E) lack complete form of a discharge characteristic of an extracellular recording. $(\mathrm{E})$ and $(\mathrm{F})$ exhibit both normal and abnormal forms of discharge; $(\mathrm{G})$ and $(\mathrm{H})$ exhibit normal forms of discharge from birds that survived 99 days. Courtesy of Cheng et al. J Neurobiol. 2004; 60: 197-213.

birds (Fig. 5). Reinstatement of this pathway before behavioral recovery may explain the parallel recovery of nest coo performance $[49,51]$. Finally, blocking the newborn cells blocks the recovery of nest coo behavior, a finding which supports the causal relationship between newborn neurons and recovery of lost functions [51].

\section{ACTIVITY-DEPENDENT ADULT NEUROGENESIS AND RECOVERY OF BEHAVIOR}

With the basic recovery timeline established, we next examined external (environmental and social) factors that might intervene in the process of recovery. The first question we addressed is whether housing conditions during recuperation affect the outcome of behavioral recovery. Our initial study of this question illustrated the advantage of (post-lesion males) being housed with the same mate (female) compared to being housed in solitude [49]. We then addressed whether the "healing" affect of a mate is specific to the female gender [51]. Finally, experiments were designed to identify the characteristics of "healing" promoting females. The most recent study on this topic highlights the principle of activity-dependent effect [53]. Simply put, the effect of an external factor (environmental or social) on recovery is contingent upon whether the external factor is involved in stimulating (i.e. engaging) newborn neurons during various stages of their incorporation into the specific circuitry responsible for a behavior. In our case, when newborn neurons, located in a previously damaged network of coo-specific responsive neurons, are activated or engaged, their synaptic connections to the circuit are presumably consolidated, which in turn leads to the survival of the newly arrived neurons and development of a functional network supporting nest coo behavior.

In studies completed in 2006-2007, we showed that lesioned males do not recover well when placed alone in the cage, nor if caged with another male. Lesioned males housed with female mates consistently fare better [49-51]. The data up to then suggested that the nature of the cage mate was the deciding factor, but it remained unclear which characteristics of this stimulus accounted for the differential effect. To address this question, we compared the effects of housing the lesioned male with their female mate or with a new female. In the latter situation, we introduced a new female every three days to block formation of bonding. We suspected that the bonding status of a female might have played a role in the earlier studies where the experimental males were always returned to their mates after surgery. Traditionally, a bird's bonding status to a mate is defined upon completing one successful breeding cycle. We recently established that the bond status (at least in the ring dove) can be reliably assessed by expression of the ZENK gene which encodes a zinc-finger-containing transcriptional regulator and can be rapidly activated in the brain [54]. This enables us to determine a dove's bond status without relying exclusively on breeding history. Zenk data was therefore used to determine the bonding status of female mates and new females in this study.

Pair bonding evolved as a reproductive strategy to ensure reproductive success. We predicted that the male housed with its female mate would recover better since bonding is critical for this species to maintain viable breeding cycles, and therefore the female mate would harbor higher value. Contrary 


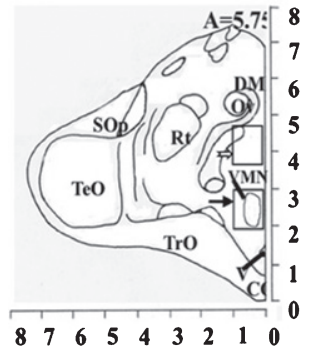

(A): Recording area Counting area

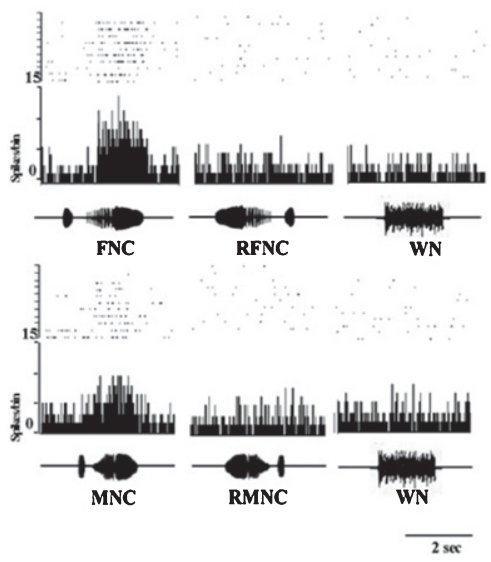

(D): Female nest-coo specific unit
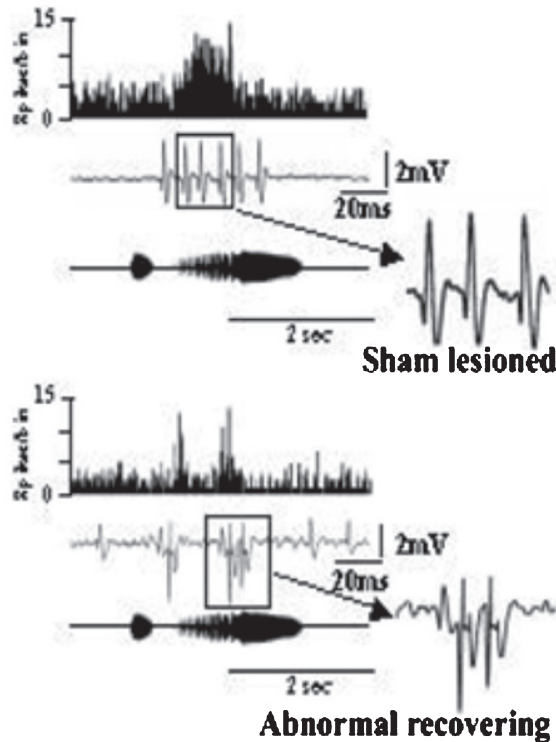
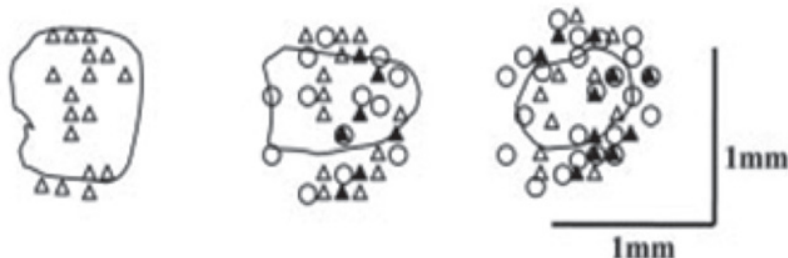

\section{Short survival Medium survival Long survival 36 days 43-71 days}

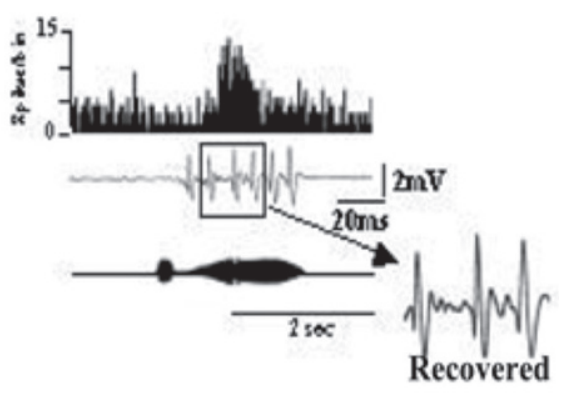

(C): Discharge patterns of units in the VMN

Fig. 4. Newborn Neurons Exhibiting Female Nest-Coo Specific Properties 3 months Postlesion. A summary of newborn neurons assuming electrophysiological properties of acoustic neurons uniquely sensitive to female coo stimulation over the course of recovery after electrolytic lesion to the VMH in the ring dove. (A) lesion site, (B) distribution of recording sites and double-labeled cells: sites not responsive (open triangle), responsive to nest coo stimulation (filled triangle), and BrdU/NeuN_double-labeled cells (open circle), (C) electrophysiological properties of a newborn neuron comparing to that from an intact brain of the same region, (D) as in intact brain, these newborn neuron respond only to female coo stimulation. Courtesy of Cheng et al. 2004; J Neurobiol. 60: 197-213.

to our prediction, the results showed that the male housed with a new female every three days recovered significantly faster, and more importantly, these males recovered within 4 weeks compared to the 8 weeks we had observed in earlier studies. Analysis of behavioral data for both males and females revealed the previously unsuspected role of male behavior in the differential pace of recovery. When exposed to a new female, the male significantly engaged or attempted to engage a new female more than their pair-bonded female, even though there was no difference in the level of female behavior between the groups to account for the male behavior; in fact, the bonded female tended to exhibit a higher level of courtship behavior at week 3 after her mate was returned from surgery. In other words, by attempting to court a new female by nest coo, the male engages in activity-dependent neural synaptic events, thereby successfully completing recruitment of functional newborn neurons.

In a subsequent study, we sought to replicate the findings and using a generalized linear mixed model, we analyzed the level of nest coo and newborn neurons at weeks 4 and 8 between bonded and nonbonded groups. This study would allow us to validate or discredit the notion that rapid behavioral recovery is made possible through the earlier appearance of newborn neurons, which we had long hypothesized 

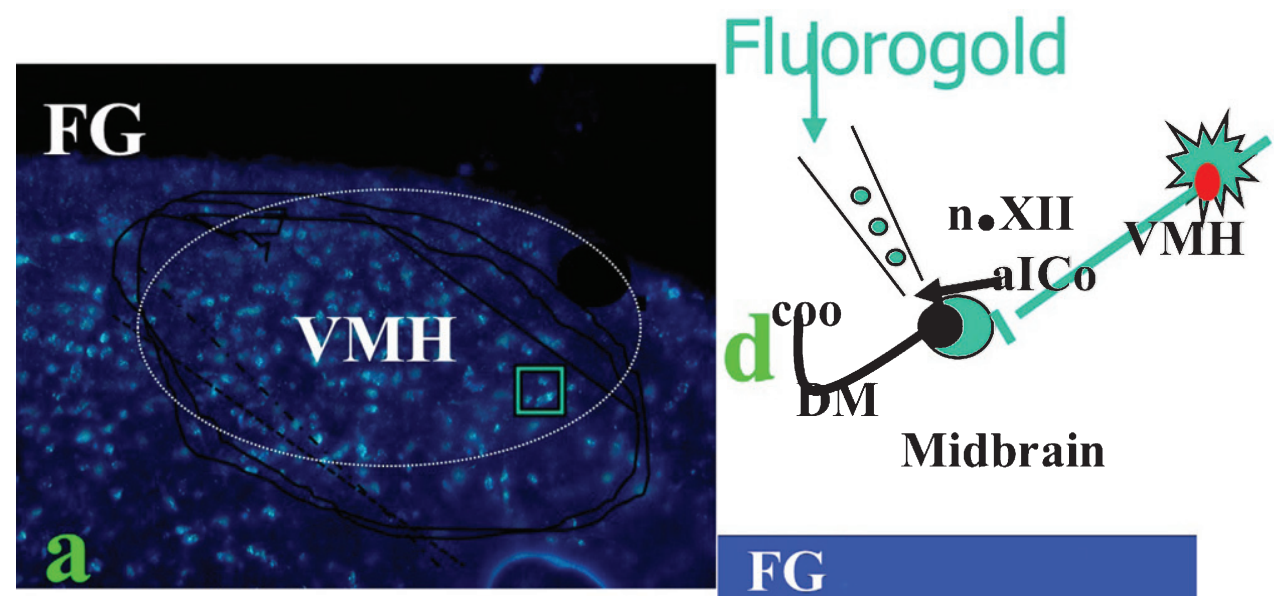

Midbrain
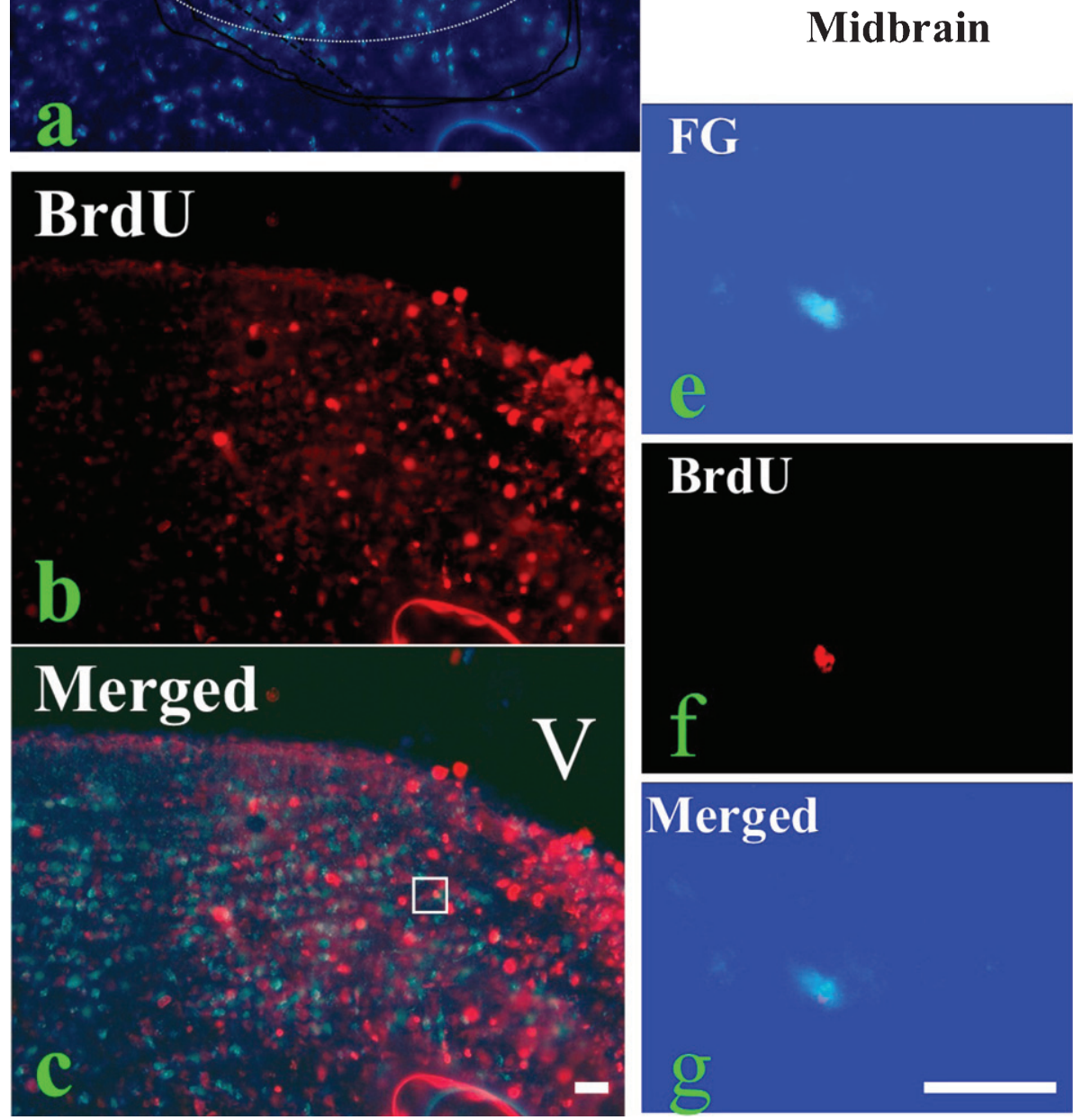

Fig. 5. Projection Neurons Born After Hypothalamic Lesion. Fluorogold (FG) was injected into the anterior ICo of the midbrain at 2 or 8 weeks after VMH lesion. Newborn midbrain (ICo)-projection neurons were detected in the VMH 8 weeks after lesion [schematically shown in (d)]. VMH was discernible with numerous retrograde FG cells (a,e). Newborn cells were labeled with BrdU (b,f). Some projection neurons were double-labeled with BrdU (c,g). V 1/4 third ventricle. Scale bar 1/4 30_m. [Color figure can be viewed in the online issue, which is available at http://www.intersciencewiley.com. Courtesy of Chen et al, 2006; J Neurobiol. 66: 537-551.

as the underlying mechanism for behavioral recovery. The results replicated the earlier study that the males housed with new females recovered nest coo levels sooner (week 4). Specifically, males housed with new females exhibited higher levels of newborn neurons and nest coo behavior at week 4 than when housed with their female mates [53]. This differential performance persisted until week 8 . The finding strengthens our conclusion that the males reinstate nest coo behavior sooner and better when exposed to a novel stimulus (a new female) during recuperative period. The new females' "healing power" lies in 
their ability to invoke the male's biological temptation to court or engage fresh females, thereby physiologically engaging the circuitry containing the newly arrived neurons, and consolidating their connection to the circuitry and survival of the new neurons. This hypothesis is consistent with numerous studies indicating the role of excitatory inputs in the form of electrical activities in developmental processes [55-57].

\section{RATE OF RECOVERY}

The conclusion that male courtship behavior after brain damage can be restored sooner than 8 weeks prompted us to revisit our older data, specifically our 2004 work in which we recorded normally evoked discharges of newborn neurons of birds kept for up to 78-99 days. We detected seemingly incompatible results between this earlier study and the later study. The later study consistently suggests that 8 weeks time is sufficient to see behavioral recovery, whereas results of the 2004 study suggest that normal neuronal discharge related to LH output in response to female nest coo stimulation does not begin until around 60 days and more typically beginning around 78-99 days [52].

Two factors could contribute to the apparent inconsistency. Firstly, behavioral recovery measured in the studies suggesting an 8-week recovery timeline was limited to measurement of courtship behavior, and did not include egg-laying behavior. It is plausible that the recovery of egg-laying behavior does not appear until 78-99 days after brain lesion. Secondly, in the 2004 study, the majority of birds were returned to stock or solitude cages after brain surgery [52], in other words, these birds were not exposed to positive, facilitative external factors as in the later studies in which birds were returned to cages containing female mates. In essence, the speed of recovery of function depends to a degree on the nature of the function.

\section{ACTIVITY-DEPENDENT SYNAPTOGENESIS IN LEARNING AND MEMORY}

The "use it or lose it" principle is evident in the hippocampus, a region which has been widely studied in recent years. Progenitor cells in the subgrandular zone of the detate gyrus migrate into the granular layer and mature rapidly, extending axons to the CA3 region of the hippocampus. Several studies in rodents have shown how environmental and behavioral factors can modulate neurogenesis and affect hippocampal-dependent learning and memory [58-61]. One study did cast some doubt on the role of neurogenesis in hippocampaldependent memory [62], possibly because not all hippocampal-dependent learning tasks engage in the hippocampal circuitries exclusively. The fact remains that more hippocampal neurons survive after certain hippocampal-dependent tasks are learned compared to when non-hippocampal tasks are learned $[60,62,63]$.

The finding that young newly generated neurons show a low threshold for induction of synaptic formation in mice [64] provides one tantalizing explanation for what transpires during the coding process of the recruitment of new neurons into the circuit. While the factors responsible for circuit integration and survival are likely to be complex and numerous, our systematic ring dove studies described above have identified the activity-dependent synaptogenesis as one viable underlying mechanism for the successful recruitment of functional newborn cells. Activitydependent synaptic event can also be activated by sensory input during development. For example, in song birds, before a song is learned, tutor song exposure drives pre-motor spiking activity within HVC (a sensorimotor interface for song). This process is suppressed by an inhibition mechanism once the song is learned [65]. Similar mechanisms may also apply to vision-mediated motor learning in adult born young neurons. In the ring dove, female exposure before new neurons establish firm synaptic connections in the nest-coo network may have driven network activity within VMH sensorimotor neurons for nest coo behavior. We speculate that compared to the bonded (familiar) female, the mere presence of a fresh (new) female is a more potent stimulus in driving new neurons arriving at the nest-coo network, thereby facilitating synaptogenesis. Future study should be able to tease apart the relative contributions of sensory and motor engagement. Whether by sensory or motor engagement, crucial to our hypothesis is the role of network activity in driving synaptic consolidation in the recruitment and survival of new neurons. Recent studies of molecular mechanisms provide further support for an activity-dependent mechanism. Activity-dependent transcription in the intracellular signaling of neurons, e.g., response to stimuli creates a new class of RNA (eRNA), thought to regulate synaptic development [66, 67]. 


\section{CONCLUSION}

Aging adults generally take longer to learn and remember new tasks, in part because the encoding process (solidifying use of the circuit) takes longer to initiate and to complete. The popular advice for aging adults to be more mindful and to exercise the brain with mental activities is presumably to engage the related circuit more intensely. As we have shown there is scientific data from multiple species to support this recommendation.

In summary, the "use it or lose it" concept is strikingly evident in our analysis of the functionality of the nervous system in the adult ring dove. This phenomenon continues throughout the life span of a living animal and may be most critical for an aging population when a sedentary lifestyle compounds the vicious cycle of declining use and declining functionality.

\section{ACKNOWLEDGMENTS}

This work was supported by the Rutgers Research Fund. I would like to thank John Franchak for technical assistant.

\section{CONFLICT OF INTEREST}

The authors have no conflict of interest to report.

\section{REFERENCES}

[1] SfN NR-15-08. Sole use of impaired limb improves recovery in spinal cord injury. Society for Neurosci News Release 9/16/2008.

[2] Maier IC, Baumann K, Thallmair M, Weinmann O, Scholl J, Schwab ME. Constraint-induced movement therapy in the adult rat after unilateral corticospinal tract injury. J Neurosci. 2008;28(38):9386-403. doi:10.1523/JNeurosci.169708.2008

[3] Nash CE, Mickan SM, Del Mar CB, Glasziou PP. Injured limbs recover better with early mobilization and functional bracing than with cast immobilization. In: Resting injured limbs delays recovery: A systematic review. J Fam Pract. 2004;53:706-12. doi:10.2106/JBJS.8705.ebo3

[4] Tillerson JL, Caudle WM, Reveron ME, Miller GW. Exercise induces behavioral recovery and attenuates neurochemical deficits in rodent models of of Parkinson's Disease. Neuroscience. 2003;119(3):899-911. doi:10.1016/S03064522(03)00096-4

[5] Wolpaw JR, Tennissen AM. Activity-dependent spinal cord plasticity in health and disease. Annu Rev Neurosc. 2001;24:807-43. doi: 10.1146/annurev.neuro.24.1.807

[6] Frisbie DD, Al-Sobayil F, Billinghurst RC. Kawcak CE, McIlwraith $\mathrm{CW}$. Changes in synovial fluid and serum biomarkers with exercise and early osteoarthritis in horses.
Osteoarthr Cartilage. 2008;16:1196-204. doi: http://dx.doi. org/10.1016/j.joca.2008.03.008

[7] van den Boom R, van de LEST CHA, S. Bull S, Brama PAJ, van Weeren PR, Barneveld R. Influence of repeated arthrocentesis and exercise on synovial fluid concentrations of nitric oxide, prostaglandin E2 and glycosaminoglycansin healthy equine joints. Equine Vet J. 2005;37(3):250-56. doi: $10.2746 / 0425164054530740$

[8] Jay GD, Waller KA. The biology of lubricin: Near frictionless joint motion. Matrix Biol. 2014;39:17-24. doi:10.10 16/j.matbio.2014.08.008

[9] Harzsch S, Dawirs R, Neurogenesis in the developing crab brain: Post embryonic generation of neurons persists beyond metamorphosis. J Neurobiol. 1996;29:384-98.doi: 10.1002/(SICI)1097-4695(199603)29:3<384::AID-NEU9 $>3.0 . \mathrm{CO} ; 2-5$

[10] Doetsch F, Scharff C. Challenges for brain repair: Insights from adult neurogenesis in birds and mammals. Brain Behav Evolut. 2001;58:306-22. doi:10.1159/000057572

[11] Arvidsson A, Colin T, Kirik D, Kokaia Z, Lindvall O. Neuronal replacement from endogenous precursors in the adult brain after stroke. Nat Med. 2002;8:963-70. doi:10. 1038/nm747

[12] Eriksson PS, Perfilieve E, Bjork-Eriksson T Alborn AMH, Nordborg C, Peterson DA, Gate FH. Neurogenesis in the adult human hippocampus. Nat Med. 1998;4:1-5.

[13] Ernst A, Frisen J. Adult neurogenesis in humans-common and unique traits in mammals. PloS Biol. 2015;13:1-12. Doi:10.1371/joumal.pbio.1002045

[14] Gage FH. Neurogenesis in the adult brain. J Neurosci. 2002;22:612-3

[15] Alvarez-Buylla A, Pardal R, Garcia-Verdugo JM, Mateo AS, Merchant-Larios H. Primary neural precursors and intermitotic nuclear migration in the ventricular zone of adult canaries. J Neurosci. 1998;18:1020-37

[16] Sohur U, Emsley JG, Mitchell BD, Macklis JD. Adult neurogenesis and cellular brain repair with neural progenitors, precursors and stem cells. Phil Trans R Soc Soc. 2006;361:1477-97. doi:10.1098/rstb.2006.1887

[17] Cheng MF. Hypothalamic neurogenesis in the adult brain. Front Neuroendocrine. 2013;34:167-78. http://dx.doi.org/ 10.1016/j.yfrne.2013.05.001

[18] Lee DA, Bedont JL, Pak T, Wang H, Song J. MairandaAngulo A, Takiar V, Charibhumi V, Balordi F, Takebayashi H, Aja S, Ford E, Fishell G, Blackshaw, S. Tanycytes of the hypothalamic median eminence form a dietresponsive neurogenic niche. Nat Neurosci. 2012;15:700-4. Doi:10,1038/nn.3079

[19] Kokoeva MV, Yin H, Flier JS. Neurogenesis in the hypothalamus of adult mice:potential role of energy balance. Science. 2005;310:679-83. doi: 10.1126/science. 1115360

[20] Gould E, Reeves AJ, Graziano MS, Gross CG. Neurogenesis in the new neocortex of adult primates, Science. 1999;286:548-52. Doi:10.1126/science.286.5439.548

[21] Bernier PJ, Bedard A, Vinet J, Levesque M, Parent A. Newly generated neurons in the amygdala and adjoining cortex of adult primates. Proc Natl Acad Sci USA. 2002;99:11 464-11 469. Doi:10.1073/pnas.172403999

[22] Zhao M, Momma S, Delfani K, Carlén M, Cassidy R, Johansson CS, Brismar H, Shupliakov O, Frisén J, Janson AMH. Evidence for neurogenesis in the adult mammalian substantia nigra. Pro Natl Acad Sci USA. 2003;100:792530. doi: 10.1073/pnas.1131955100

[23] Lindvall O, McKay R. Brain repair by cell replacement and regeneration. Proc Natl Acad Sci. 2003;100 (13):7430-1. doi:10.1073/pnas.1332673100 
[24] Frielingsdorf $\mathrm{H}$, Schwarz K, Brundin P, Mohapel P, No evidence for new dopaminergic neurons in the adult mammalian substantia nigra. Proc Natl Acad Sci USA. 2004;101:10 177-10 182. Doi:10.1073/pnas.040122 9101

[25] Shihabuddin LS, Horner PJ, Ray J, Gage FH. Adult spinal cord stem cells generate neurons after transplantation in the adult dentate gyrus. J Neurosci. 2000;20:8727-35

[26] Arvidsson A, Collin T, Kirik D, Kokaia Z, Lindvall O. Neuronal replacement from endogenous precursors in the adult brain after stroke. Nat Med. 2002;8:96370:doi:10.1038/nm747

[27] Parent JM, Vexler ZS, Gong C, Derugin N, Ferriero DM. Rat forebrain neurogenesis and striatal neuron replacement after focal stroke. Ann Neurol. 2002;52:802-13. doi:10.10 02/ana.10393

[28] Teramoto T, Qiu J, Plumier J-C, Moskowitz MA. EGF amplifies the replacement of parvalbumin-expressing striatal interneurons after ischemia. J Clin Invest. 2003;111:1125-32. doi:10.1172/JCI200317170

[29] Bédard A, Lévesque M, Bernier PJ, Parent A. The rostral migratory stream in adult squirrel monkeys: Contribution of new neurons to the olfactory tubercle and involvement of the antiapoptotic protein Bcl-2. Eur $\mathrm{J}$ Neurosci. 2002;16(10):1917-24. doi: 10.1046/j.1460-9568. 2002.02263

[30] Nakatomi H, Kuriu T, Okabe S, Yamamoto S, Hatano O, Kawahara N, Tamura A, Kirino T, Nakafuku M. Regeneration of hippocampal pyramidal neurons after ischemic brain injury by recruitment of endogenous neural progenitors. Cell. 2002;110:429-41. doi:10.1016/S0092-8674(02) 00862-0

[31] Margavi SS, Leavitt BR, Macklis JD. Induction of neurogenesis in the neocortex of adult mice. Nature. 2000;405:951-55. doi : 10.1038/35016083

[32] Cao J, Cheng MF. Lesion induced new neuron incorporation in the cells in the adult hypothalamus. Brain Res. 2002;943:80-92.

[33] Gould E, Tanapat P. lesion-induced proliferation of neuronal progenitors in the dentate gyrus of the adult rat. Neuroscience. 1997;80:427-36.

[34] Yoshimura S, Takagi Y, Harada J, Teramoto T, Thomas SS, Weber C, Bakowska JC, Breakfield XO, Moskowitz MA. FGF-2 regulation of neurogenesis in adult hippocampus after brain injury. Proc Natl Acad Sci. 2001;98:5874-879. doi:10.1073/pnas.101034998

[35] Xiong Y, Mahmood A, Chopp M. Angiogenesis, neurogenesis and brain recovery of function following injury. Curr Opin Investig Drugs. 2010;11:298-308.

[36] Goldman SA, Nottebohm F. Neuronal production, migration, and differentiation in a vocal control nucleus of the adult female canary brain. Proc Natl Acad Sci USA. 1983; 80:2390-4.

[37] Scharff C, Kirn JR, Grossmann MR, Macklis JD, Nottebohm F. Targeted neuronal death affects neuronal replacement and vocal behavior in adult songbirds. Neuron. 2000;25:481-92.

[38] Nottebohm F. Neuronal replacement in adulthood. Ann NY Acad Sci.1985;457:143-61.

[39] Cheng MF. The role of vocal self-stimulation in female responses to males: Implications for state-reading. Horm Behav. 2008;53:1-10.

[40] Cheng MF. Interaction of lighting and other environmental variables on activity of hypothalamo-hypophyseal-gonadal system. Nature. 1976;263:148-59. doi:10.1038/263148a0
[41] Bernstein PL, Zuo M, Cheng MF. Social condition affects the courtship behavior of male ring doves with posterior medial hypothalamic lesions. Behav Neural Biol. 1993;59:120-5.

[42] Gibson M, Cheng, MF. The neural mediation of estrogen dependent courtship behavior in the female ring dove. J Comp Physiol Psychol. 1977;93:855-67.

[43] Durand S, Tepper J, Cheng MF. The shell region of the nucleus ovoidalis: A subdivision of the avian auditory thalamus. J Comp Neurol. 1992;323:495-518. doi: 10.1002/cne. 903230404

[44] Cheng MF, Peng J. Reciprocal talk between the auditory thalamus and hypothalamus: An antidromic study. NeuroReport. 1997;8:653-8.

[45] Cheng MF, Peng JP, Johnson P. GnRH neurons preferentially respond to female nest coo stimulation: Demonstration of direct acoustic stimulation of luteinizing hormone release. J Neurosci. 1998;18:5477-89.

[46] Cheng MF. Female cooing promotes ovarian development in ring doves. Physiol Behav. 1986;37:371-4. doi:10.1016/0031-9384(86)90248-9

[47] Cheng MF, Desiderio C, Havens M, Johnson A. Behavioural stimulation of ovarian growth. Horm Behav. 1988;22:388402.

[48] Cheng MF. Vocal Self-stimulation: From the ring dove story to emotion-based vocal communication. In: Slater PJ, Rosenblatt JS, Snowdon CT, Roper TJ, Naguib M, editors. Advances in the Study of Behavior. Elsevier Academic Press. 2003;33:309-53. doi:10.1038/263148a0

[49] Chen G, Bonder E, Cheng MF. Lesion induced neurogenesis in the hypothalamus is involved in behavioral recovery in adult ring doves. J Neurobiol. 2006;66:537-51. doi: 10.1002/neu. 20247

[50] Cheng MF, Alexander K, Zhou S, Bonder, Chuang LS. Newborn GnRH neurons in the adult forebrain of the ring dove. Horm Behav. 2011;60:94-104. doi: 10.1016/j.yhbeh. 2011.03.008

[51] Chen G, Cheng MF. Inhibition of Lesion-Induced Neurogenesis impaired behavioral recovery in adult ring doves. Behav Brain Res. 2007;177:358-63. doi:10.1016/j.bbr. 2006.11.041

[52] Cheng MF, Peng JP, Chen G, Bonder EM. Functional restoration of acoustic units and adult-generated neurons after hypothalamic lesion. J Neurobiol. 2004;60:197-213. doi10.1002/neu.20014

[53] Dios A. Pair bonding:what mediates its formation and maintenance? Rutgers, The State University of New Jersey, 2015 https://rucore.libraries.rutgers.edu/rutgers-lib/47648/

[54] Dios AM, Alexander K, Hanson S, Cheng MF. Specific neural representation for a conceptual set of behavior: Pair bonding. Res Rep Biol. 2013;4:39-40. doi: http://dx.doi.org/ 10.2147/RRB.S56210

[55] Balazs R, Hace N, Jørgensen OS. Stimulation of NMDA receptor has a trophic effect on differentiating cerebellar granule cells. Neurosc Lett. 1988;87:80-6. doi:10.1016/03 04-3940(88)90149-8

[56] Brenneman DE, Eiden DE. Vasoactive intestinal peptide and electrical activity influence neural survival. Proc Natl Acad Sci. 1986;83:1159-62.

[57] Lipton SA. Blockade of electrical activity promotes the death of mammalian retinal ganglion cells in culture. Proc Natl Acad Sci USA. 1986;83:9774-8.

[58] Kemperman G, Kuhn HG, Gage FH. More hippocampal neurons in adult mice living in an enriched environment. Nature. 1997;386:493-5. doi:10.1038/386493a0 
[59] van Praag H, Kempermann G, Gage FH. Running increases cell proliferation and neurogenesis in the adult mouse dentate gyrus. Nature Neurosci. 1999;2:266-70.

[60] Gould E, Beylin A, Tanapat P, Reeves A, Shors TJ. Learning enhances adult neurogenesis in the hippocampal formation. Nat Neurosci. 1999;2:260-65. doi: 10.1038/6365, 2002;12:578-84. doi: 10.1002/hipo.10103

[61] Deng W, Aimone JB, Gage FH. New neurons and new memories: How does adult hippocampal neurogenesis affect learning and memory? Nat Rev Neurosci. 2010;11:341-50. doi: $10.1038 / \mathrm{nrn} 2822$

[62] Shors TJ, Townsend DA, Zhao M, Kozorovitskiy Y, Gould E. Neurogenesis may relate to some but not all types of hippocampal-dependent learning. Hippocampus. 2002;12:578-84. Doi:10.1002/hipo.10103

[63] Bruel-Jungerman E, Rampon C, Laroche S. Adult hippocampal neurogeneis, synaptic plasticity and memory: Facts and hypothesis. Rev Neurosci. 2007;18:93-114. doi: 10.1515/REVNEURO.2007.18.2.93
[64] Bischofberger J. Young and excitable: New neurons in memory networks. Nat Neurosc. 2007;10:273-75. Doi:10.1038/ nn0307-275

[65] Vallentin D, Kosche G, Lipkind D, Long MA. Inhibition protects acquired song segments in zebra finches. Science. 2016;351:267-71. Doi:10.1126/science.aad3023

[66] Lam MT, Li W, Rosenfeld MG3, Glass CK. Enhancer RNAs and regulated transcriptional programs. Trends Biochem Sci. 2014;39:170-82. doi: 10.1080/15592294.2015.1034 416

[67] West AE, Greenberg ME. Neuronal activity-regulated gene transcription in synapse development and cognitive function. Cold Spring Harb Perspect Biol. 2011;3:a005744. doi: 10.1101/cshperspect.a005744 